\title{
Funding Evidence: The National Institute of Neurological Disorders and Stroke Clinical Trials Program
}

\author{
Bernard Ravina, ${ }^{*}$ Scott Janis, ${ }^{*}$ Julianna Keleti, ${ }^{\dagger}$ and John M. Marler* \\ *National Institute of Neurological Disorders and Stroke, National Institutes of Health, Bethesda, Maryland 20892; and \\ ${ }^{\dagger}$ KAI Research, Inc., Rockville, Maryland 20852
}

\begin{abstract}
Summary: The goal of the National Institute of Neurological Disorders and Stroke (NINDS) Clinical Trials Program is to foster clinical trials that will provide the evidence needed to inform clinical care. The NINDS currently supports clinical research in over 150 neurological disorders. The rapid pace of preclinical discovery and the diversity of neurological diseases, however, present challenges for clinical trials. There is a growing number of potential interventions to be tested in clinical trials. The NINDS Clinical Trials program is therefore exploring ways of making drug selection for clinical trials more evidence-based. Additionally, NINDS supports pilot clinical trials that focus on the timely and efficient testing of agents to
\end{abstract}

determine if resource-intensive comparative efficacy trials are warranted. In concert with the National Institutes of Health Roadmap, NINDS is planning to expand clinical trials infrastructure. This infrastructure is intended to enable the conduct of clinical trials for rare diseases and diseases without previous trials experience and facilitate the recruitment of a broad range of participants. Rigorous programs to select agents, and design and monitor clinical trials will encourage the efficient use of this clinical trials infrastructure and will ensure that NINDSfunded studies meet the highest scientific and ethical standards. Key Words: National Institutes of Health, clinical trials, clinical research.

\section{INTRODUCTION}

The mission of the National Institute of Neurological Disorders and Stroke (NINDS) is to lessen the burden of neurological disease. Toward that end, NINDS supports clinical research in over 150 neurological disorders. The role of the Clinical Trials program at NINDS is to facilitate and oversee extramural clinical trials that will provide evidence to inform important clinical decisions. The Clinical Trials program has the following three main strategies to meet this goal: 1) foster clinical research and clinical trials across the spectrum of adult and pediatric neurological disorders, 2) facilitate the timely and efficient translation of promising basic research into clinical studies, and 3) ensure that clinical trials in neurology meet the highest standards of scientific and ethical conduct. Here we describe the efforts of NINDS to implement these strategies and thereby provide high-quality evidence to inform clinical care.

Address correspondence and reprint requests to Bernard Ravina, M.D., National Institute of Neurological Disorders and Stroke, Bethesda, MD 20892-9525. E-mail: ravinab@ninds.nih.gov.

\section{CLINICAL TRIALS IN PROGRESS}

The NINDS is currently funding 142 extramural clinical trial protocols, representing a broad array of adult and pediatric neurological disorders. Twenty-one of these are phase I studies (human pharmacology in healthy volunteers or patients), 94 are phase II studies (exploration of the activity of the intervention in the disease population), and 27 are phase III (comparative efficacy). In total, these trials are investigating interventions for 40 different neurological diseases. Figure 1 illustrates the distribution of NINDS-funded clinical trials by disease category. Nearly $75 \%$ of the studies currently funded by the Institute focus on five disease categories, including some of the more common neurological disorders such as cerebrovascular disease, disorders of movement, brain and spinal trauma, seizure disorders and epilepsy, and CNS neoplasms. The remaining 25\% of the NINDS-funded trials are evenly spread across 10 disease categories, which include CNS infections, demyelinating diseases, developmental disorders, disorders of the autonomic nervous system, disorders of the peripheral nervous system and motor neuron disorders, genetic diseases, headache, muscular disorders, pain, and sleep disorders. Overall there are currently 10 


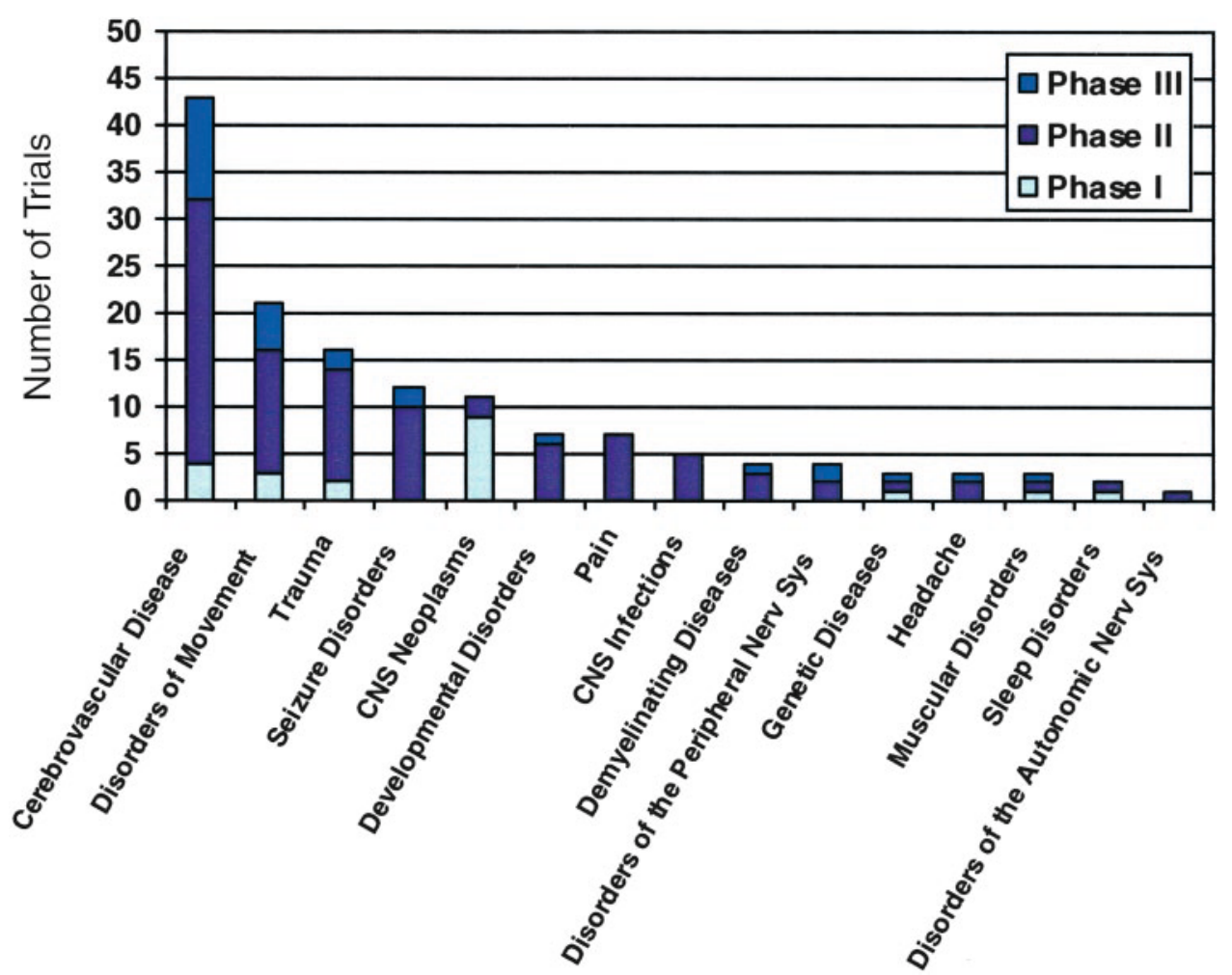

FIG. 1. Distribution of NINDS-supported phase I, phase II, and phase III clinical trials across neurological disease categories.

phase III studies in these areas, including phase III trials in multiple sclerosis (MS), attention deficit hyperactivity disorder, amyotrophic lateral sclerosis (ALS), sickle cell anemia, and periodic paralysis.

There are multiple factors that determine the patterns for NINDS support of clinical trials. These include the availability of potential targets and therapeutic opportunities in the particular field, the burden of illness of the disorder, the quality of protocols submitted for review, and whether other sources of funding are available. For example, although headache is one of the most common neurological disorders, there is strong commercial interest in supporting trials and, hence, relatively fewer NINDS trials. The goal of the NINDS clinical trials program is therefore not to equalize the number of clinical trials in different disease categories, but to provide the support and resources needed to conduct high-quality clinical trials for any neurological disease when significant therapeutic opportunities arise that would not otherwise be addressed.

\section{THE FIRST TRANSLATIONAL STEP: FROM PRECLINICAL WORK TO CLINICAL TRIALS}

Breakthroughs in biomedical sciences including genomics, proteomics, and molecular biology have advanced and broadened the potential for interventions to improve human health. NINDS and other NIH institutes continue to invest in preclinical therapy development through drug screening, animal model testing, and efforts to provide the preclinical information needed for clinical testing. ${ }^{1-3}$ The challenge for the clinical research infrastructure is to translate these findings into clinical studies. A disconnect between the support of preclinical and clinical research could undermine the promise of preclinical discoveries. ${ }^{4,5}$ This concern has been widely recognized and the barriers encountered in conducting clinical research have been codified in a report from the Institute of Medicine Clinical Research Roundtable. ${ }^{5}$

The NINDS clinical trials program is currently addressing two important issues in translational research and clinical trials: 1) the identification and prioritization of interventions for testing and 2) the design and implementation of pilot clinical trials that efficiently select agents for further study in comparative efficacy trials.

Clinical trial planning begins with the selection of an agent for clinical testing. The path by which particular agents make it into clinical trials, however, is often unclear. Potential therapies may wait for years before moving into clinical trials and many promising interventions may never reach clinical trials at all. There are several reasons for the failure of an agent to gain entry into pilot clinical trials, including but not limited to a lack of communication between preclinical and clinical researchers and the availability of competing interventions. Additionally, the peer review process by mandate evaluates proposals and interventions on their own merits 
TABLE 1. Power Calculations in Pilot Clinical Trials in Neurology Published in 2002

\begin{tabular}{lcccc}
\hline Primary Aim & $\begin{array}{c}\text { Power Calculation/ } \\
\text { All Studies }\end{array}$ & $\begin{array}{c}\text { Power Calculation/ } \\
\text { Phase I }\end{array}$ & $\begin{array}{c}\text { Power Calculation/ } \\
\text { Phase II }\end{array}$ & $\begin{array}{c}\text { Power Calculation/ } \\
\text { Pilot }\end{array}$ \\
\hline Preliminary efficacy/activity & $20 / 80$ & $0 / 2$ & $17 / 36$ & $3 / 42$ \\
Efficacy & $4 / 20$ & - & $2 / 3$ & $2 / 17$ \\
Dose finding & $0 / 9$ & $0 / 9$ & $\overline{0}$ & $0 / 3$ \\
Safety/tolerability & $0 / 5$ & $0 / 1$ & $\overline{1}$ & $0 / 1$ \\
Feasibility & $0 / 1$ & - & $19 / 40$ & $5 / 63$ \\
Total & $24 / 115$ & $0 / 12$ & & \\
\hline
\end{tabular}

without comparison to other trials. Thus, while the agents studied in such peer-reviewed trials are meritorious, there is no prioritization to ensure that the most promising agents are tested.

NINDS is supporting ways of making drug selection more complete and robust. As part of an NINDS program to test drugs with the potential to slow the progression of Parkinson's disease (PD), NINDS established the Committee to Identify Neuroprotective Agents for Parkinson's (CINAPS) to systematically gather and evaluate information about candidate interventions. ${ }^{6}$ CINAPS was composed of clinical pharmacologists, neurologists, clinical trialists, and laboratory-based experts in neurodegeneration. The group established explicit criteria including mechanism and rationale, efficacy in animal models of PD, safety and tolerability in humans, pharmacokinetics, and preliminary evidence of activity in humans. Working with academic researchers, industry, and the lay community, CINAPS gathered information on 59 different compounds. The published literature on each compound was then used to rate these agents on the prespecified criteria. Twelve compounds with a variety of mechanisms of action were found to be good candidates for phase II-IV clinical trials assessing neuroprotection in PD. The CINAPS approach revealed several promising interventions that had been available for years but had not been fully studied. ${ }^{6,7}$ The ongoing NINDSsponsored trials, known as the Neuroprotection Exploratory Trials in PD (NET-PD), are testing four compounds identified by CINAPS.

The appeal of the CINAPS process is that it brings an evidence-based approach to drug selection. Criteria are prespecified and data on interventions are gathered from multiple sources and systematically characterized. This allows for the objective prioritization of interventions rather than relying on the most recent hypotheses or expert opinion. The approach has the added appeal of broad participation, which facilitates acceptance by investigators. A similar approach to identifying and characterizing compounds is underway in Huntington's disease (HD) (http://www.huntingtonproject.org) and will be developed for other neurodegenerative disorders.

The evidence to support interventions is only as good as the quality of trials that test them. The growing num- ber of potential interventions presents the problem of selecting agents for further study in large, phase III clinical trials. There are generally accepted design features for phase III or comparative efficacy studies. Broadly, these features include randomization, blinding, and placebo controls. Even these basic tenets may be controversial in certain trials, such as sham surgery trials for fetal tissue transplantation in PD. ${ }^{8}$ In contrast, pilot clinical trials leading to phase III studies have wide variability of aims and the application of basic design elements. In pilot or phase I-II clinical trials, the goal is not to determine if the agent "works," but to further refine the intervention-dose, timing, and method of administration-or to provide information about the intervention's activity in specific study populations, ${ }^{9,10}$ in preparation for an eventual phase III trial of efficacy. Without these studies, phase III trials are likely to fail.

The variable quality and design of pilot clinical trials in neurology can be demonstrated by a review of the published literature. To systematically assess these, a PubMed and Medline search was conducted using the terms "phase I," "phase II," or "pilot" clinical trial, and "neurological disease." In this manner, we identified 115 clinical trials of pharmacological interventions published in 2002 (Table 1). These studies were then further categorized by their stated aim, or if no primary aim was stated, this was derived from the study design by the reviewers (J.K. and B.R.). Of the 115 studies, only $21 \%$ had published power or sample size calculations. The failure to account for this basic element of study design was apparent across all five specified categories of pilot trials, including those attempting to determine efficacy or activity of the intervention. Underpowered studies or studies that are of unknown power are a widely recognized problem in clinical research. The ability to draw conclusions from these studies is limited because of high type II (false negative) error rates. Many clinical researchers regard studies with inadequate or unknown power as unethical because they expose human subjects to risks without the ability to answer the study question. ${ }^{11}$ False negatives, which increase as statistical power decreases, are of particular concern for diseases that have no existing treatments, because potentially useful compounds will be abandoned. In addition to the 
TABLE 2. Type of Control Used by Pilot Clinical Trials in Neurology Published in 2002

\begin{tabular}{lcccccc}
\hline Controls & All Studies & Dose Finding & Efficacy & Feasibility & Preliminary Efficacy/Activity & Safety/Tolerability \\
\hline Active & 8 & - & 2 & - & 4 & 2 \\
Historical & 6 & - & - & - & 6 & - \\
Negative & 7 & - & -1 & - & 6 & - \\
None & 70 & 8 & - & - & 60 & 1 \\
Placebo & 24 & 1 & 17 & 1 & 80 & 5 \\
Total & 115 & 9 & 20 & 1 & & \\
\hline
\end{tabular}

obvious problem of power and statistical inference, there is wide variation in other design aspects of pilot trials. Table 2 demonstrates the use of controls in pilot clinical trials, which ranges from no controls at all to active controls. The appropriateness of the control group determines the validity of the conclusions that can be drawn by comparison to the experimental group. Often no concurrent control group is needed in early phase studies, but if a concurrent control group is used the exact purpose and type of control needed should be carefully justified.

Recognizing the variability of and uncertainty about pilot trial design, NINDS implemented the Pilot Clinical Trials Program. This program is intended to facilitate the conduct of investigator-initiated preliminary studies that will lead to better designed, definitive, phase III trials (http://grants.nih.gov/grants/guide/pa-files/PAR-03-174. html). This program is focused on developing critical information about the intervention or study population and on using the appropriate design tools for each phase of clinical development. A notable feature of this program is an emphasis on the judicious use of concurrent or internal controls. Cancer clinical trials have used external, historical controls in pilot studies since the 1970s. ${ }^{12}$ For example, the proportion of subjects tolerating drug or responding to drug may be compared to a prespecified cutoff derived from previous studies. This design has the added benefit of allocating all patients to the intervention, which may increase the ability to determine the intervention's activity and side effects (B. Tilley, personal communication). It is expected that improved pilot clinical trials methods will more accurately identify agents that merit further study, leading to more positive phase III trials.

\section{MONITORING CLINICAL TRIALS AND PROTECTING PARTICIPANTS}

The quality of evidence coming from clinical trials depends not only on the design of the study but the actual conduct of the study. For many readers a clinical trial appears as a relatively short, single report. The actual process of information gathering that occurs throughout the trial may not be readily apparent from the final report and may be an overlooked source of random error or bias. There are numerous issues that can arise in the process of conducting a trial that are critical for the ultimate validity of that trial and for the protection of human subjects. These issues may range from errors in data collection, coding, and reporting to more systematic problems. For example, one of the most problematic issues in phase III trials is drop-out or loss to follow-up. Different drop-out rates in arms of the study may bias clinical trials because nonresponders or subjects who do not tolerate the intervention may be more likely to leave the trial than responders or those who do not experience side effects. Data Safety Monitoring Boards (DSMBs) are charged with monitoring the performance of most phase III clinical trials. They necessarily consider some of the performance and safety issues in closed sessions to avoid the risk of unblinding of the study, but these closed-session deliberations can undermine confidence in the study. Therefore, general procedures and methods for safety and performance monitoring need to be as clear and transparent to participants and researchers as the study protocol itself to preserve confidence in the integrity of the trial. ${ }^{13}$

To ensure the safety and integrity of NINDS-funded trials, NINDS continues to refine and implement procedures for monitoring all types of human subject studies by way of safety monitors, safety monitoring committees, and DSMBs (http://www.ninds.nih.gov/funding/ clinical_trials/monitoring_policies.htm). All trials to be conducted under NINDS funding are reviewed internally for risks to determine the level of monitoring required. Members of the clinical trials program work with investigators to develop a full study monitoring plan. Small studies and single-center studies may require only a safety monitor, whereas all phase III trials have independent DSMBs that report to NINDS. Monitoring clinical trials is not only a way to assure their ongoing safety and progress but to guarantee that they produce the highest quality evidence possible.

\section{CLINICAL TRIALS INFRASTRUCTURE}

Conducting successful clinical trials requires welltrained investigators and adequate infrastructure to oversee and manage the flow of data. Relative to fields such as oncology or cardiology, which have extensive net- 
works for clinical investigation, neurology has relatively fewer clinical research networks. However, clinical research infrastructure and expertise in neurological trials are increasing with the potential for therapeutic interventions. There are clearly established groups of clinical investigators with clinical trials track records in Alzheimer's disease, PD, HD, ALS, stroke, MS, and other neurological disorders. These groups vary in their cohesiveness, success in finding sources of funding, and development of methods for determining which agents they will study.

There remain, however, many neurological disease areas in which there is limited or no clinical trials experience. For this reason, NINDS is planning to develop clinical trials infrastructure that will broadly serve neurological diseases and will facilitate cooperation among different members of the clinical research enterprise. The diversity of neurological diseases would seem to call for independent groups of clinical researchers, but shared resources will enable different subfields to learn from each others' experiences and will facilitate patient recruitment, broad-based participation of investigators, and the use of common data elements, data collection tools, and clinical practices. This approach may be particularly helpful for rare diseases and will make clinical trials operations for these diseases more economically feasible.

There are currently two clinical trials infrastructure projects that are early in the planning stages. The first is a network for conducting pilot clinical trials that will be supported by a coordinating center with the ability to design protocols and provide the operational support for early and middle development clinical trials. This network will conduct a small number of pilot clinical trials for particularly promising interventions and will focus on diseases that lack clinical trials infrastructure and need the services of the network. The second project is the Clinical Research Collaboration (CRC). The overall objective of the NINDS CRC is to facilitate the efficient execution of NINDS-sponsored clinical research to develop and test more treatments more expeditiously. Unlike the pilot network, the CRC will not function in the traditional role of a coordinating center for a clinical trial. Rather, the CRC will assist NINDS-supported, investigator-initiated trials with recruitment by involving the broadest possible range of patients and academic and community-based physicians. As envisioned, neurologists would recruit and follow patients through access to multiple clinical research protocols funded through investigator-initiated clinical trials. By including a broad range of participants including women and minorities, the CRC will facilitate recruitment and enhance the generalizability of clinical trials. By including a wide range of physicians from various practice settings, the CRC will broaden the experience with study interventions and may thereby help to overcome the second translational step, the implementation of research discoveries in clinical care.

These networks portend the changing nature of the clinical research workforce. In fact "Reengineering the Clinical Research Enterprise" is a cornerstone of the NIH Roadmap as envisioned by NIH director Elias Zerhouni. ${ }^{14}$ The expansion and integration of clinical research infrastructure are major aims of the NIH roadmap and are part of the broader NIH plans for reshaping clinical research infrastructure. This process will include efforts to train clinical researchers for the future, better integrate clinical research networks to include a broader range of patients and health care professionals, harmonize research regulatory requirements across government agencies, and facilitate data sharing. The clinical research infrastructure of the future will have a greater diversity of participants and more integration of data.

\section{THE ROLE OF NINDS IN DEVELOPING THERAPIES}

NINDS funds clinical trials for many different neurological diseases and is expanding the infrastructure to support clinical trials, but what is the actual role of NINDS in the broader context of developing treatments and evidence to inform clinical care? NIH and other government agencies including the Food and Drug Administration support research in diseases that typically fall below the market threshold of pharmaceutical companies. These include rare diseases like Huntington's disease, ataxias, myasthenia gravis, and many others. However, even for more common diseases like stroke, government funded studies can play a critical role. For example, the NINDS funded trials of recombinant tissue plasminogen activator (t-PA) led to the approval of this drug for the treatment of acute stroke. ${ }^{15}$ These positive trials were conducted while several trials of thrombolytic agents with time windows of $6 \mathrm{~h}$ or greater failed to reach statistical significance. ${ }^{16}$ The NINDS trials were different from previous trials in their use of a $3 \mathrm{~h}$ time window from onset of stroke for inclusion and in the use of a global statistical test for the outcome measures. ${ }^{15}$ The manufacturer's only role in these trials was to supply drug and placebo. The t-PA trials demonstrate that NINDS and academic investigators can conduct rigorous and innovative trials that play a pivotal role in developing drugs in cooperation with industry.

Rarely does a single phase III trial answer all important therapeutic questions and the need for evidence does not stop after drug or device approval. Clinical care often demands additional studies of treatment strategies or more practical effectiveness studies to determine if the results from trials extend to less controlled settings. The pharmaceutical industry, which profits from developing new agents, cannot always be expected to be the sole 
sponsor of postmarketing studies for new indications. They may, however, collaborate with the NIH by providing the agent or other resources. NINDS is currently supporting trials in several diseases for new uses of an FDA-approved intervention. For example, the VA and NINDS are collaborating in a study to compare deep brain stimulation - of the globus pallidus and subthalamic nucleus - to best medical management of PD. The secondary analysis will compare the two surgical locations. A phase IV (postmarketing) clinical trial in MS will compare combinations of immune modulating therapies from different manufacturers to single use of these therapies.

There are some potential interventions that are owned by companies that are not interested in or capable of making the significant investments required for studying new indications. For example, some generic drugs and food supplements would not come to clinical trials without NIH support. The food supplement Coenzyme Q10 is one of the more promising interventions for several neurodegenerative diseases including ALS, HD, and PD. A sickle cell anemia trial is examining the role of transfusions, a nonproprietary intervention. The pharmaceutical marketplace does not encompass all interventions and cannot be expected to answer all clinically important questions. NIH-funded trials play an important role in developing new treatments and in determining how best to use those treatments in clinical care.

\section{CONCLUSIONS}

Clinical trials are resource- and labor-intensive. The experience and infrastructure needed for conducting trials are not uniformly developed in neurology. However, it is imperative that clinical research keep pace with preclinical discoveries and practical clinical questions if patients are to reap the benefits of progress in the biomedical sciences. The NINDS Clinical Trials Program is focusing efforts on bringing the pipeline of emerging therapies into trials in an efficient manner, by thoroughly evaluating interventions and providing clinical research infrastructure and training. The infrastructure will facil- itate the translation of preclinical discoveries into clinical trials and will aid the conduct of trials for rare diseases. Efficient use of these resources through well designed, well monitored studies will yield the greatest chance of developing important treatments for neurological diseases.

\section{REFERENCES}

1. Finkelstein R, Miller T, Baughman R. The challenge of translational research-a perspective from the NINDS. Nat Neurosci 5:1029-1030, 2002.

2. Heemskerk J, Tobin AJ, Bain LJ. Teaching old drugs new tricks. Trends Neurosci 25:494-496, 2002.

3. Heemskerk J, Tobin AJ, Ravina B. From chemical to drug: neurodegeneration drug screening and the ethics of clinical trials. Nat Neurosci 5:1027-1029, 2002.

4. Nathan DG, Wilson JD. Clinical research and the NIH-A report card. N Engl J Med 349:1860-1865, 2003.

5. Sung NS, Crowley WF, Genel M, Salber P, Sandy L, Sherwood $\mathrm{LM}$ et al. Central challenges facing the national clinical research enterprise. JAMA 289:1278-1287, 2003.

6. Ravina B, Fagan SC, Hart RG, Hovinga CA, Murphy DM, Dawson TM, Marler JR. Neuroprotective agents for clinical trials in Parkinson's disease: a systematic assessment. Neurology 60:12341240, 2003.

7. Wheatley K, Stowe RL, Clarke CE, Hills RK, Williams AC, Gray R. Evaluating drug treatments for Parkinson's disease: how good are the trials? BMJ 324:1508-1511, 2002.

8. Freed CR, Greene PE, Breeze RE, Tsai WY, DuMouchel W, Kao R, Dillon S, Winfield H, Culver S, Trojanowski JQ, Eidelberg D, Fahn S. Transplantation of embryonic dopamine neurons for severe Parkinson's disease. N Engl J Med 344:710-719, 2001.

9. Choi DW. Exploratory clinical testing of neuroscience drugs. Nat Neurosci 5:1023-1025, 2002.

10. Chabner BA, Boral AL, Multani P. Translational research: walking the bridge between idea and cure-seventeenth Bruce F. Cain Memorial Award Lecture. Cancer Res 58:4211-4216, 1998.

11. Halpern SD, Karlawish JH, Berlin JA. The continuing unethical conduct of underpowered clinical trials. JAMA 288:358-362, 2002.

12. Herson J. Predictive probability early termination plans for phase II clinical trials. Biometrics 35:775-783, 1979.

13. Wittes J. Behind closed doors: the data monitoring board in randomized clinical trials. Stat Med 12:419-424, 1993.

14. Zerhouni E. Policy forum: the NIH roadmap. Science 302:63-72, 2003.

15. Tissue plasminogen activator for acute ischemic stroke. The National Institute of Neurological Disorders and Stroke rt-PA Stroke Study Group. N Engl J Med 333:1581-1587, 1995.

16. Hinchey JA, Benesch C. Thrombolytic therapy in patients with acute ischemic stroke. Arch Neurol 57:1430-1436, 2000. 